\title{
Debatiendo las metodologias participativas: Un proceso en ocho saltos
}

Discussing participatory methodologies: A process in eight jumps

\author{
Javier Caballero Ferrándiz \\ Colaborador Red CIMAS \\ Pedro Martín Gutiérrez \\ Universidad de Valladolid \\ Tomás R. Villasante \\ Universidad Complutense de Madrid
}

pmartingu@hotmail.com (ESPAÑA)

Recibido: 28.022018

Aceptado: 18.06 .2019

\section{RESUMEN}

Para considerar verdaderamente implicadas y transformadoras a las metodologías participativas se plantea un diseño de 8 saltos basado en el caso de Entrevías-El Pozo (Madrid). La articulación de las metodologías participativas, como paradigma, con otras técnicas como las reuniones de grupo o los sociogramas, suponen la implicación de los sujetos en el diseño y la implementación de la propia investigación, así como en su organización orientada a la acción. En este diseño se trata de jugar con las nociones de "abrir para cerrar" y "cerrar para abrir" (Villasante, 1994) que permiten diversos procesos, a través de técnicas como la auto-reflexión, la negociación inicial, el mapa estratégico, la escucha activa con multi-lemas, los talleres de creatividad social, la priorización de propuestas, la estructura auto-organizativa y el desborde. Para ello se realiza una descripción del proceso de Entrevías-El Pozo siguiendo el diseño antes comentado. Este caso sirve de referente para el desarrollo metodológico de los 8 saltos. 


\title{
PALABRAS CLAVE
}

Diseño metodológico, metodologías participativas, técnicas de investigación, técnicas de intervención.

\begin{abstract}
To consider truly involved and transformative participatory methodologies a design of 8 jumps is proposed based on the case of Entrevías-El Pozo (Madrid). The articulation of participatory methodologies, as a paradigm, with other techniques such as group meetings or sociograms, they imply the involvement of the subjects in the design and implementation of the research itself, as well as in its action-oriented organization. In this design it is about playing with the notions of «open to close» and «close to open» (Villasante, 1994) that allow various processes, through techniques such as self-reflection, initial negotiation, the map strategic, active listening with multi-slogans, social creativity workshops, the prioritization of proposals, the self-organizational structure and the overflow. To do this, a description of the Entrevías-El Pozo process is made, following the aforementioned design. This case serves as a reference for the methodological development of the 8 jumps.
\end{abstract}

\section{KEY WORDS}

Methodological design, participatory methodologies, research techniques, intervention techniques.

\section{INTRODUCCIÓN}

En los "Ayuntamientos del Cambio" (y en otros), así como en varios movimientos sociales (vecinales, de salud, urbanísticos, juveniles, etc.) hay un debate sobre los nuevos planes integrales, comunitarios o de barrios, y las participaciones que han presentado. Además, hay que añadir otro debate actual sobre los Presupuestos Participativos (bastantes de tipo digital y sin auto-diagnósticos descentralizados y deliberados) para que se construyan criterios desde la ciudadanía, para la auto-reglamentación de los mismos. Hay un trasfondo ideológico-político en todo ello sobre las causas y consecuencias de unas y otras estrategias participativas. Aquí vamos a tratar de reflexionar más desde el ángulo metodológico propiamente dicho, basándonos en experiencias concretas en las que hemos participado, sacando algunas consecuencias comparativas.

También nos enfrentamos a los debates sobre metodologías participativas, IAP, metodologías activistas, socio-análisis, socio-praxis, etc., que hemos po- 
dido mantener, entre otros, en el Foro Ibérico de Metodologías Participativas ${ }^{1}$, en el Congreso sobre Participación y Democratización del Conocimiento, de Cartagena de Indias ${ }^{2}$ y en la Red Sentipensante ${ }^{3}$. En estos espacios hemos podido ir avanzando en la construcción colectiva, en las autocríticas y las aportaciones de las diferencias de cada estrategia metodológica. Por ejemplo, hace unos años escribíamos y practicábamos con referencia a 6 saltos diferenciales en estas metodologías, y ahora (tras un debate que hemos reiniciado en la Red CIMAS ${ }^{4}$ ) vamos a presentar 8 saltos que consideramos muy importantes para poder considerar más implicadas y transformadoras a las metodologías de participación.

En las últimas décadas hemos incorporado aportaciones de varias tradiciones emancipadoras, a fin de delimitar un marco teórico abierto, pero diferenciándonos de algunas trampas y atajos que aparecen en los propios procesos participativos. Como resumen y para poder encuadrar de donde partimos adjuntamos un cuadro reactualizado de las posiciones, autores y movimientos sociales a los que nos remitimos ${ }^{5}$. Aportamos las principales referencias que nos han servido de base en la construcción de la Socio-Praxis.

${ }^{1}$ Madrid, abril de 2016 (http://www.foroparticipacion.net/)

${ }^{2}$ Cartagena de Indias, Colombia, junio de 2017 (http://arnawebsite.org/conferences/cartegena-colombia-2017/)

${ }^{3}$ Rosario, Argentina, noviembre de 2018 (https://globaleduca.hypotheses.org/498)

${ }^{4}$ Vid.: http://www.redcimas.org/

5 Para un mayor detalle y comprensión de estos enfoques nos remitimos a Villasante (2007) 


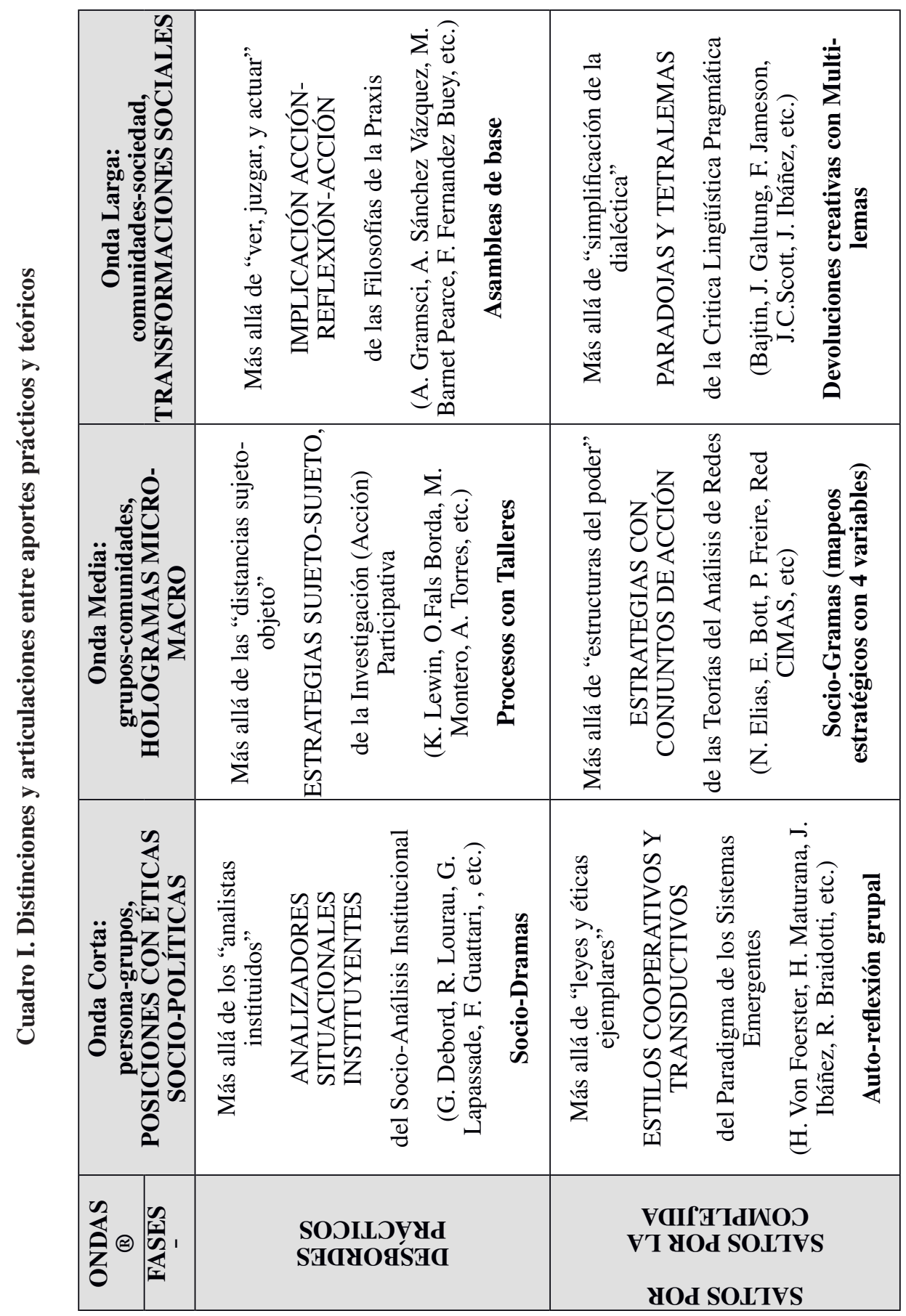

EMPIRIA. Revista de Metodología de Ciencias Sociales. N. ${ }^{4} 4$ septiembre-diciembre, 2019, pp. 21-45. ISSN: 1139-5737, DOI/ empiria.43.2019.25350 


\begin{tabular}{|c|c|c|c|c|c|c|c|c|c|c|c|}
\hline 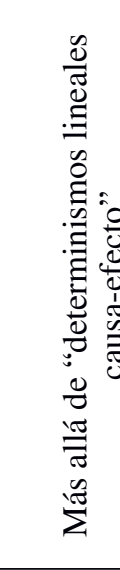 & 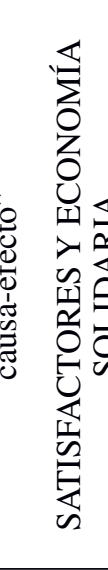 & 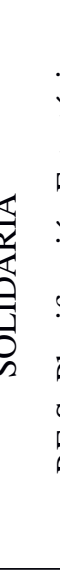 & 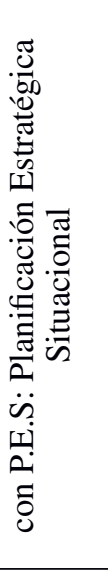 & 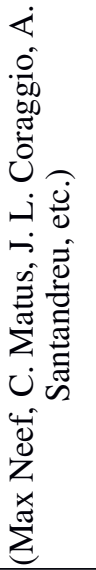 & 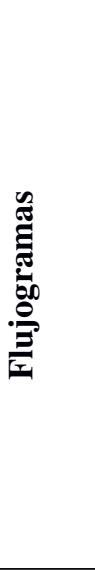 & 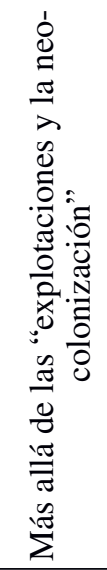 & 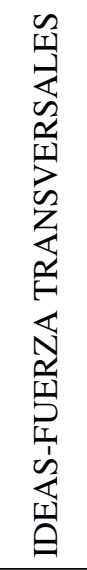 & 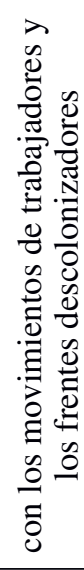 & 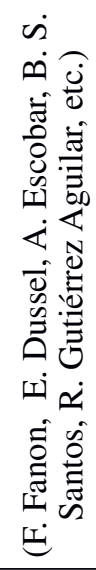 & 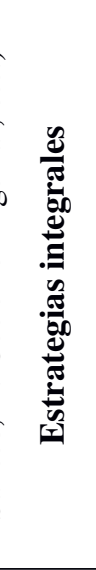 & \\
\hline 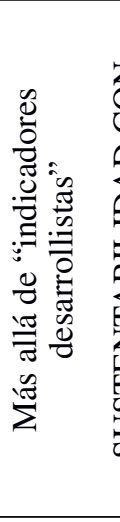 & 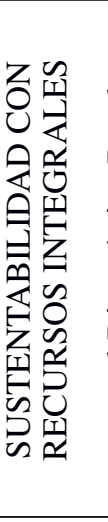 & 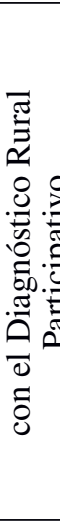 & 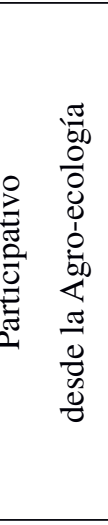 & 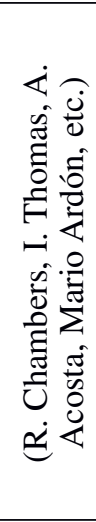 & 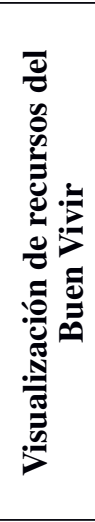 & 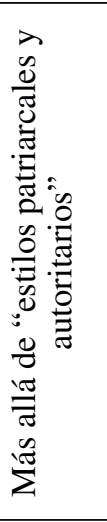 & 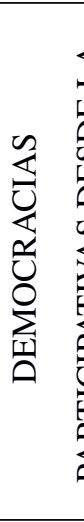 & 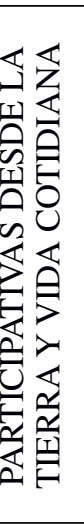 & 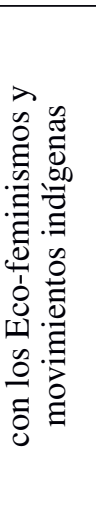 & 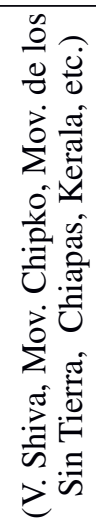 & 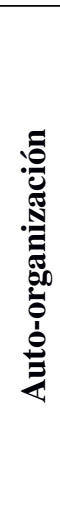 \\
\hline 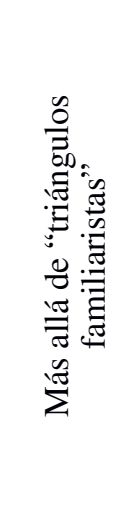 & 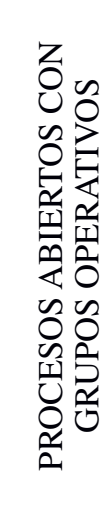 & 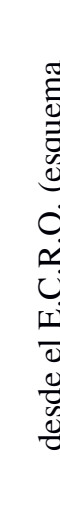 & 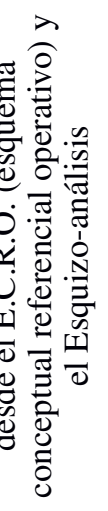 & 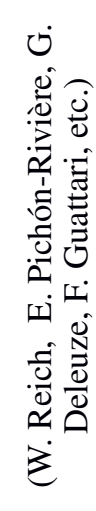 & 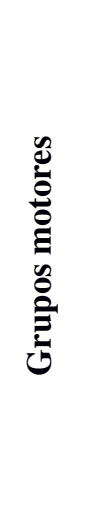 & 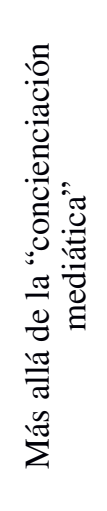 & 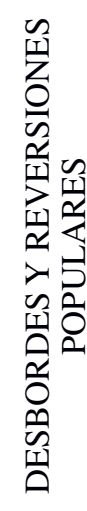 & 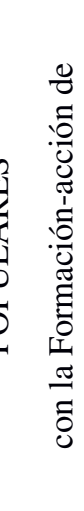 & 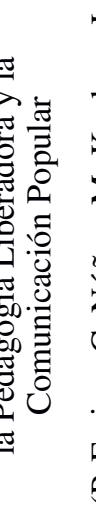 & 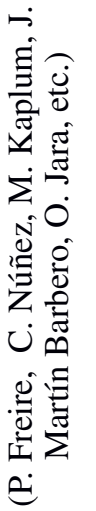 & 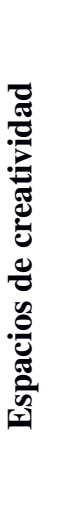 \\
\hline \multicolumn{6}{|c|}{ 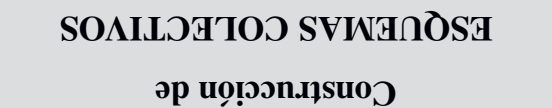 } & \multicolumn{6}{|c|}{ XX OTDIS SOLNHIWIAOW } \\
\hline
\end{tabular}

EMPIRIA. Revista de Metodología de Ciencias Sociales. N. 44 septiembre-diciembre, 2019, pp. 21-45. ISSN: 1139-5737, DOI/ empiria.43.2019.25350 


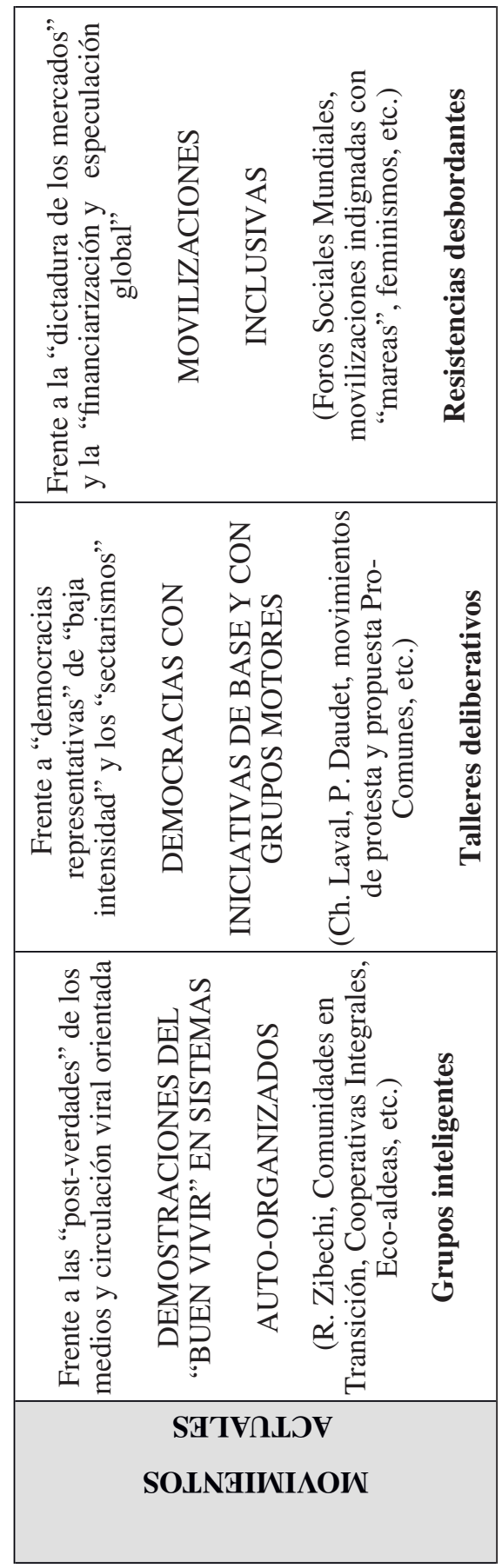

EMPIRIA. Revista de Metodología de Ciencias Sociales. N. 44 septiembre-diciembre, 2019, pp. 21-45. ISSN: 1139-5737, DOI/ empiria.43.2019.25350 


\section{INVESTIGACIÓN-ACCIÓN-PARTICIPATIVA: UNA CUESTIÓN DE PARADIGMA, NO DE TÉCNICA.}

Partamos de la Investigación Acción Participativa (IAP). Es común entre quienes se dedican a la intervención social y a la IAP la reticencia a "lo cuantitativo". Y más en concreto, a la encuesta y a la sistematicidad de "recetas metodológicas milagrosas" (Colectivo IOÉ, 1996: 39). Buena cuenta de ello viene inspirada por la crítica, ya clásica, de Jesús Ibáñez a la perspectiva distributiva (2015 [1986]) y la defensa de una perspectiva dialéctica. Sin embargo, como comentan Javier Callejo y Antonio Viedma (2006: 181) en su capítulo sobre IAP, "lo importante no es el instrumento, sino la posición ética que mantenga el investigador que lo utilice". En este sentido, lo que determina a la IAP es una posición sobre su intervención en la realidad, el por qué y para qué de la investigación más allá del desarrollo técnico y metodológico (Red CIMAS, 2015).

Tomar a la IAP y otras metodologías participativas desde un enfoque, paradigma o epistemología alternativa, no solo como metodología o como técnica, implica que "la IAP no sería tanto una forma distinta de investigar [...] como una especial articulación de diversas prácticas de investigación ya conocidas (encuestas, entrevistas, grupos, etc.) con el objetivo de una intervención dirigida al cambio" (Callejo y Viedma, 2015: 296). Esta diferencia entre paradigmas y metodologías/técnicas se basa en la noción, ya desarrollada por Guba y Lincoln (2002: 113), de que "el uso de métodos tanto cualitativos como cuantitativos puede ser apropiado para cualquier paradigma de investigación. De hecho, las cuestiones de método son secundarias frente a las de paradigma, que definimos como el sistema básico de creencia o visiones del mundo que guía al investigador, ya no sólo al elegir los métodos, sino en formas que son ontológicamente y epistemológicamente fundamentales".

Por lo tanto se reconoce que "los instrumentos técnicos que se utilizan en estos casos tampoco son inocentes" (Colectivo Ioé, 1996: 37), y que lo fundamental es tener en cuenta el para qué y por qué se utilizan. No solo la IAP, como metodología, está relacionada con la praxis: tanto las metodologías cualitativas, como cuantitativas, afectan a la realidad y la transforman. La IAP como paradigma afecta al resto de técnicas (encuesta, entrevista, etc.) a partir de la implicación de los propios sujetos de la investigación en su diseño, como sujetos-objeto: "la identificación de los problemas (y soluciones), que no parte de los expertos, y el grado de implicación de los sujetos observados, tanto entre sí como en el propio proceso de investigación y acción" (Callejo y Viedma, 2015: 295). Es así como se puede utilizar una encuesta que ha sido elaborada por el propio Grupo motor (Colectivo Ioé, 1996), o cómo una sesión de grupo puede servir para priorizar algunas de las propuestas recogidas en los anteriores talleres (Red CIMAS, 2015). Es "una investigación para la acción concreta" (Callejo y Viedma, 2015: 296). 


\section{DISEÑO DE UNA SOCIO-PRAXIS, "CERRAR PARA ABRIR" $Y$ “ABRIR PARA CERRAR” CON 8 SALTOS DIFERENCIALES.}

En este sentido, recuperamos los términos de Tomás Villasante (1994: 415) de "cerrar para abrir" y "abrir para cerrar" los procesos como aproximaciones metodológicas para la construcción de uno de tipo socio-praxico. La intención, además, es la de avanzar respecto a las metodologías participativas y "su nivel de instrumentación técnica poco desarrollado" (Colectivo Ioé, 1989: 6). La premisa de este diseño de implicación-acción está en la complementariedad por deficiencia desarrollada por Alfonso Ortí (1986). Este planteamiento vincula a la metodología cualitativa con el contexto de descubrimiento, y a la metodología cuantitativa con el contexto de justificación. Mediante éstos dos últimos términos nos hacemos eco de Jesús Ibáñez (1985) y su referencia a Hans Reichenbach. Este autor relaciona el "contexto de descubrimiento" con aquellas "actividades exploratorias, que contribuyen a la generación de hipótesis", y el "contexto de justificación" con aquellas capaces de la verificación o falsación de una hipótesis (Cea D’Ancona, 2012: 34). Sin ánimo de centrarnos en la verificación de hipótesis, sino en la organización de la acción, el fundamento de esta vinculación entre metodologías estaría en que la metodología cualitativa aparece como una necesaria "apertura" a los significados y el sentido de los actores en la realidad social, mientras que la metodología cuantitativa como una forma de concretizar y delimitar esta realidad. El proceso de "abrir para cerrar" es extensivo, mientras que el proceso de "cerrar para abrir" es intensivo.

Sin embargo, y tal y como plantea Villasante, a los procesos de "abrir para cerrar" y "cerrar para abrir" se añaden los de "simetría" y "asimetría" entre los actores. Es importante tener en cuenta estos dos últimos conceptos pues son los que permiten la articulación entre el grupo motor y los participantes en talleres a lo largo de un proceso. Comenta que: "epistemológicamente es aceptar una táctica asimétrica (partir de lo que hay: expertos, promotores, bases), para una estrategia de mejor simetría (siempre relativa), cerrar contrastes entre sectores diferentes (precisar las contradicciones entre grupos y con las bases, triángulos de relaciones en las comunidades), para abrir la re-construcción de la red existente (negociar, participar, etc.)" (Villasante, 1994: 416). Todo proceso plantea un cierre (asimétrico) y una apertura (simétrica) con la que hay que saber jugar. Pues "en la cuanti-distributiva sus efectos son la asimetría (cierra), en la cualiestructural la simetría es táctica pero la asimetría es estratégica y en la IAPdialéctica la simetría (abre)." (Villasante, 1994: 416). La socio-praxis pretende también distinguirse de la IAP, que sólo abre, sin precisar cómo.

En este sentido, hay unos 8 "saltos" que nos parece que suelen diferenciar un proceso (hecho con "socio-praxis" y más garantías metodológicas) de otros hechos con más voluntad y buena intención que conocimientos de los problemas que suelen surgir. Pasamos a proponer unas aclaraciones y distinciones sobre los sentidos que le damos a estos "saltos" para que no se puedan confundir solo con fases o etapas de un proceso. Proponemos unas aclaraciones y distinciones, por- 
que en cada etapa/salto hay novedades de enfoques, de acciones y de productos a conseguir:

a) Dentro de un proceso hay fases y etapas secuenciales, que pueden alterar su proceder, pero que deben recorrerse para avanzar a sistemas más avanzados de conciencia social, de auto-organización y resultados operativos. Los saltos en este sentido no solo son cumplimentar las fases en el orden que sea, sino construir resultados tanto internos al grupo motor como externos para la comunidad en cuestión.

b) Son también saltos de enfoques en los procesos metodológicos, porque no basta con dinamizar o facilitar solo con buenas intenciones, con voluntarismo basista, sino que parece preciso establecer distinciones sobre lo que supone cada "acción", para qué sirve y para qué no sirve. Los 8 saltos que se proponen no son cualquier dispositivo técnico, sino aquellos que nos parecen que marcan unas distancias con las formas no críticas de enfocar los procesos participativos.

c) Además son "acciones" y "productos", pues nos parecen ambas cosas a la vez. Por ejemplo, la auto-reflexión es un enfoque (de base epistemológica), es una acción (mediante un socio-drama o un transecto, p.ej.), y es un producto (la forma de hacer el plan de dinamización con un grupo motor, p.ej.). Solo en la medida en que se dan estas circunstancias podemos considerar que se produce un salto metodológico (respecto a metodologías más convencionales, que no se plantean esto, y respecto a procesos que hacen técnicas sin justificar por qué o para qué, las acciones sus productos).

Vamos a enunciar cada "salto" y, al final de cada uno, para concretar, aparecerá, de manera telegráfica, lo que supone su Enfoque diferencial con otras metodologías, de qué tipo de Acciones puede precisar y qué Productos pretenden lograr.

\subsection{La auto-reflexión del equipo y del grupo motor:}

Un primer salto respecto a otras prácticas metodológicas es la "auto-reflexión" del equipo o el grupo motor que pone en marcha un proceso. En muchas ocasiones no hay tiempo para hacer este pre-requisito que nos parece importante. Se aduce que en el movimiento ya se tiene claro lo que se quiere, o que la administración tiene unos plazos y un presupuesto cerrado. Se da por supuesto que quienes quieren hacer participación son buena gente y que están dispuestos a escuchar y tomar las decisiones con la gente. Pero bajo esta capa de buenas intenciones pueden y suelen esconderse muchos prejuicios de los que los actores no somos conscientes. Todos tenemos pre-juicios que no controlamos, por lo que es preferible hacer algún ejercicio que nos permita reconocerlos, de forma que se puedan controlar y no interferir en el momento menos oportuno.

Cuando hay tiempo para hacer una formación-acción previa solemos incluir algún ejercicio (deriva, transectos, sociodrama, juego de rol, línea del tiempo, etc.) que permita sacar a la luz, desde la visión de otras personas, planteamientos previos que pudieran dificultar que el proceso sea realmente más horizontal entre 
los actores. Prejuicios de la gente de ciudad hacia la rural, de los técnicos hacia la gente sin estudios, entre etnias y culturas, hacia quien no dedica tiempo a estas tareas, etc. Es difícil que cada cual nos demos cuenta de nuestros prejuicios, por eso es importante que desde otras culturas nos puedan ver y recomendar contención en expresiones o actitudes. Y si esto se puede hacer desde el principio y con cierto grado de informalidad, escuchando a las otras personas, caminando o jugando, pues suele salirnos mejor.

La construcción de un "grupo motor" mixto, o sea de personas más técnicas y personas más "convivenciales" del lugar o el sector que estén interesadas en el proyecto concreto nos parece un factor de avance muy interesante. No las autoridades administrativas ni los dirigentes de los movimientos, sino más bien gente no tan ocupada, que pueda dedicar un tiempo real a hablar con la gente, a ser informantes locales desde la vida cotidiana, desde los rumores y los entramados del tejido social concreto. Los tiempos y los lenguajes de un grupo así ya dan un primer aterrizaje al proyecto y lo pueden ir salvando de academicismos de los técnicos y de voluntarismos y precipitaciones de la propia gente -sobre todo de aquellos dirigentes o profesionales que dicen que "ya lo saben todo"-.

Enfoque: Quién observa al observador. Acción: Desde fuera del equipo o con dispositivos de auto-reflexión. Producto: auto-limitación de algunos prejuicios para sesgar menos el proceso.

\subsection{La negociación inicial y el plan de trabajo.}

Distinguimos en la "negociación inicial" otro salto que marca todo el proceso, pues con las partes que lo inician se determinan los objetivos, tiempos totales, metodologías concretas, y condiciones de recursos, seguimiento, etc. La voluntad política y administrativa suele ser en principio muy generosa, hasta que hay que concretar la toma de decisiones real, los presupuestos y los tiempos reales, etc. por lo que suele ser importante dejar un escrito firmado por las partes, dejando claro el protagonismo compartido. Suele ser bueno que se constituya una Comisión de Seguimiento, que se reúna de tiempo en tiempo, a la que el "grupo motor" más cotidiano rinde cuentas. Desde la "negociación inicial" ya sabemos cuáles son los límites que puede haber en el proceso, al menos los iniciales, pues siempre aparecen otros imprevistos, y por tanto saber hasta dónde se puede juzgar participativo o no lo que se va a acometer.

Las reuniones pueden ser una buena ocasión para conocerse entre diversos sectores y grupos. El apego emocional y el apoyo mutuo es el pegamento entre el grupo base (afectivo) y el grupo de trabajo (de tarea). Es necesario reconocerse y tratarse para llegar a ser un grupo inteligente (Cembranos y Medina, 2003). Es bueno ser grupo base antes que grupo de trabajo, pero también hace falta ser grupo de trabajo si no se quiere caer en el mero grupo emocional. Dado que el grupo base trabaja sobre lo que sentimos, lo que pensamos, qué inquietudes tenemos, qué miedos y desconfianzas; el grupo de trabajo se plantea el qué podemos hacer. Es necesario articular estas dos formas de interacción grupal para 
la acción: ambas, en sus correspondientes proporciones, están presentes en cada momento del proceso.

En el grupo de trabajo "no se trata, por consiguiente, de averiguar qué es un grupo, de conocer su sustancia (o esencia) y sus fundamentos, sino más bien de saber qué puede un grupo" (Vercauteren, Mouss Crabbé y Müller, 2010: 157). Es importante pensar el grupo como potencia y como grupo de trabajo capaz de desarrollar recursos y potencialidades para organizarse.

Enfoque: Construcción colectiva desde las instancias iniciales. Acción: Constitución de Comisiones de Seguimiento y de Grupos Motores. Producto: Concretar la metodología al caso, y plan de dinamización.

\subsection{Mapas estratégicos y conjuntos de acción.}

Un tercer salto metodológico se refiere a que el grupo motor pueda elaborar un "mapeo de relaciones entre actores", un "mapa estratégico" (P. Freire) o "socio-grama con conjuntos de acción" (Red CIMAS, 2015). No se trata solo de identificar en un mapa local la situación de los actores, ni los puntos degradados o saludables de un barrio. Eso puede estar bien y lo hacemos. Se trata de situar sobre un cuadrante de poder real y de afinidad cultural al tema que nos interesa dónde están los diversos sectores o grupos o departamentos administrativos que intervienen. Con los que se puede contar como "afines" o bien como "diferentes", dentro de los mismos intereses. Aparte estarán la mayoría de la población "ajena”, y mucho más lejos y en contra los "opuestos”. Con estos gráficos podemos construir los "conjuntos de acción" pertinentes, y elaborar alianzas o estrategias participativas.

Sirven además para tener una "muestra relacional" de cuáles son los "nudos" de las redes clave de la vida cotidiana, por donde fluye la información básica y se reproducen las culturas en que se mueve la gente. No es una técnica para hacer en grandes talleres abiertos, sino para orientación del grupo motor, para saber a quién escuchar desde un pensamiento reticular. Es decir, que para escuchar a toda la gente seguramente basta con tener conversaciones amplias y distendidas con una docena o dos, según el tamaño del proceso, de los nudos de los entramados de vida cotidiana en donde se mueve y reproduce la gente sus ideas y acciones. Construir una estrategia participativa no es simplemente llamar a que venga la gente a donde estamos nosotros, pues como mucho solo vendrán los "afines", es saber a dónde hay que ir a escuchar la diversidad de posiciones posibles, y distribuirse la tarea.

Enfoque: Reconocer la complejidad de relaciones en los entramados sociales (clase, afinidad cultural, organización, miedos y confianzas) Acción: mapeo estratégico o sociograma. Producto: Muestra relacional y "conjuntos de acción" para alianzas viables en el proceso. 


\subsection{Escuchar más allá de los dilemas dominantes.}

La escucha activa con "multi-lemas" es otro salto que viene de lo que acabamos de plantear, pero en nuestro caso esta escucha tiene unas características especiales. Se trata de ir más allá de las peleas y dilemas dominantes que solemos encontrar. Se suele pensar que lo más repetido en las conversaciones es lo que nos da una información en la que apoyarnos para asentar las mayorías. Podemos "saturar las opiniones" con una o dos decenas de entrevistas, escuchas y talleres, pero lo habitual es que surjan varias posiciones contradictorias. No se trata de saber qué opina la mayoría con un cuestionario, pues suele ser una posición superficial. Mucho más real es saber cuántas opiniones están enfrentadas en la comunidad o sector, donde suele haber dos que se disputan la hegemonía entre lo dicho públicamente, y suele haber además otras dos o tres adicionales, que complejizan y profundizan el campo -los que no se dicen a la primera, y que solo en la confianza de un proceso aparecen-.

Llamamos "multi-lemas" a lo que Johan Galtung (2004) llama "pentalemas" y que otros autores llaman "tetra-lemas" (Ibáñez, 1985) con tal de no dejar la escucha encerrada en "dilemas". En varios de los casos señalados el grupo motor o incluso el equipo, con materiales dispersos, recogidos aquí y allá, pudo identificar las posiciones que superaban los "dilemas dominantes" y en esa medida devolverlos a la gente para establecer un auto-diagnóstico complejo. Muchas veces se destacan las posiciones mayoritarias de cada tema, como en los ejercicios académicos: "el barrio opina que..." y ya está, pero no es tan simple pues suele haber posturas controvertidas. Cuando hacemos este ejercicio suele suceder que posiciones minoritarias u ocultas pasan a ser posiciones emergentes en un debate, porque incluyen y superan los dilemas más repetidos. Para ello es muy importante preguntar a las preguntas y reflexionar sobre los "por qué" y "para qué".

Enfoque: Superar algunos dilemas superficiales que dice la gente, queriendo quedar bien (no suelen revelar posiciones ocultas). Acción: Talleres para la devolución de las posturas diferenciadas con multi-lemas. Producto: Deliberación de posiciones discursivas por los protagonistas y profundización en causas y en vías superadoras.

\subsection{Coproducir con talleres de creatividad social.}

Llegamos a los "talleres de creatividad social", que pueden darse después de varios meses o al año de haber empezado. En varios de los casos estas devoluciones de lo escuchado a la gente se hacen en diversos momentos, tanto en talleres con poca gente como en un acto más amplio (en este caso dividiendo a la gente en grupos temáticos en el propio acto). Se pueden hacer "multilemas" con las frases recogidas, y/o "flujogramas" con las posiciones que los participantes aportan en su grupo o en reuniones amplias, u otros dispositivos de priorización participativa (Red CIMAS, 2015). 
Lo primero es que la gente sienta que se tiene en cuenta su opinión y que pueden decidir, pues la información es suya, es un acto ético. Lo segundo es que puedan verificar lo que haya salido de las escuchas realizadas, tanto posiciones mayoritarias como otras emergentes. Luego profundizar en las causas manifiestas y ocultas en grupos pequeños; pasar en algún momento a relacionar e integrar unas temáticas con otras, y priorizar aquellos aspectos que la gente considera más urgentes o más importantes. La quinta cosa es diferenciar entre las propuestas de urgencia, las temáticas de fondo, y la idea-fuerza que agrupa todo el proceso. En sexto lugar se suele proponer que, en grupos mixtos, se complete la puesta en marcha de cada una de las tareas que se han señalado como prioritarias.

En la priorización de propuestas y la división del trabajo la premisa es la de que no se debe volver atrás en el proceso: volverlo a abrir, desandar lo hecho y replantearse lo dicho puede generar una frustración y una confusión sobre las posibilidades de pasar a la acción, puede comprometer el proceso: "para que no vuelva la frustración es preciso que la gente vea y compruebe que sus iniciativas desde la vida cotidiana pueden encontrar un cauce y llegar a realizarse" (Villasante, 2017: 180). Es importante tomar algunas propuestas, más o menos consolidadas, y empezar con ellas. Esta es la decisión estratégica que debe tomar el conjunto de participantes: en función de los posibles recursos y las demandas de la población, debe elegir una serie de propuestas para su puesta en marcha, en un "efecto demostración" que estimule.

Enfoque: Reflexividad de "segundo grado" con la gente implicada, que se replantea los primeros síntomas para pasar a problemáticas mucho más profundas e integrales. Acción: Talleres o Asambleas con grupos de trabajo. Producto: un auto-diagnóstico, con prioridades y alianzas para poder pasar a la fase de actuaciones.

\subsection{Planificación de urgencia y a medio plazo.}

El objetivo es el salto a la Planificación desde abajo, desde la gente que acuda desde la diversidad de sectores que hay en cualquier comunidad. No tanto que sean muchas las personas en número, sino que se vea que hay mayores y jóvenes, varones y mujeres, de distintas culturas locales, distintas ideologías o religiones, etc. Los grupos de trabajo son para acciones concretas, que se han acordado entre los implicados, y se diversifican según lo que les interesa a cada persona o asociación. Son una manera de abrir los procesos a sectores concretos, que se sienten parte del conjunto, pero tienen sus propios objetivos y capacidad de acción. Abren el proceso a quienes no llegaron al principio y a las posiciones discursivas más variadas.

Es importante diferenciar las propuestas de urgencia, las que se pueden hacer en unos meses o un año, de las que necesitan más tiempo para ser ejecutadas. Para que la gente crea en el proceso y apoye y se implique hay que realizar cuanto antes las propuestas que se vienen demandando desde hace tiempo. Como se 
demuestra en los Presupuestos Participativos, si no hay realizaciones anuales que se puedan ver y tocar, la gente no cree en promesas, las haga quien las haga. Se trata de un test de credibilidad. Aunque no se trata de crear falsas expectativas, pero sí hay que pasar de los auto-diagnósticos a realizaciones viables. Y al tiempo una "Idea-Fuerza" o visión de futuro que sea muy movilizadora para la comunidad, que sea integral de lo que se quiere en el fondo por los diversos sectores implicados, puede dar una proyección interesante al Plan, e interpelar a las autoridades para que no puedan desconocer que han de aportar ayudas y recursos humanos.

A riesgo de pecar de reiterativos es preciso repetir la clave para que haya articulación entre sí de todos estos grupos: que se reconozcan en una Idea-Fuerza común porque la hayan construido y la sientan como propia. A este respecto cabe decir que son las personas mayores quienes más recursos de tiempo tienen, que son las que principalmente se pueden vincular al proceso de una forma más completa. Por el contrario, quienes trabajan en el sector público, por ejemplo, aunque tenga menos tiempo, podrán utilizar sus recursos de influencia para comunicar e influir. Como comenta el Colectivo Ioé (1996: 50), "así se pudo descubrir que los miembros de la corporación municipal (incluido el cartero), los maestros, el médico, la asistente social, los profesores de educación de adultos y los participantes en una tertulia de padres eran los cauces más adecuados para poner en marcha el proyecto".

Enfoque: Estilos "transductivos" como verificación empírica. Acción: Grupos de trabajo mixtos, que elaboran sus propuestas y las van poniendo en marcha. Producto: Un Plan de acción integral y sustentable.

\subsection{La capacidad auto-organizativa de la gente.}

La "estructura auto-organizativa" que construye la gente implicada es la garantía de que se puedan ir consiguiendo los resultados esperados. Por eso dudamos de aquellos procesos (como algunos planes o presupuestos participativos) que no dejan auto-organización para la toma de decisiones. Si se dejan de hacer asambleas abiertas, si un grupo reducido se relaciona con la administración y no cuenta con grupos de trabajo para temas concretos, suele suceder que el proceso decae. Por el contrario, un Plan Integral debe contar con todas las formas posibles de ampliar los conjuntos de acción; un buen indicador es el número de jóvenes y la diversidad de colectivos, dando algunos resultados iniciales e involucrando a sectores que al principio desconfían de que vaya en serio. No se trata solo de la autogestión, sino de qué se puede combinar con la cogestión con las administraciones que se precisen en cada caso. La Comisión de Seguimiento, donde pueden estar personas de la administración y portavoces de la población, puede ser una formula entre otras para la democracia participativa.

Este salto muestra hasta qué punto se ha conseguido hacer "escuela de ciudadanía" de formación-acción, que deje poso en la comunidad o sector considerado. Sin duda es una prueba que sólo se consigue en algunas ocasiones, al menos 
por las experiencias que conocemos, pero que es posible y deseable. Se necesita una presencia de estilos transductivos de hacer las cosas y la continuidad de grupos motores durante varios años. Incluso en los mejores casos, de una generación que se haya auto-educado en estas prácticas de democracias de iniciativas desde abajo. En los pueblos con cierta continuidad de estos procesos o en barrios pequeños donde se ha practicado estas metodologías, por lo que hemos señalado, es más fácil que en las grandes poblaciones a las que nos hemos referido. Por eso, partir de una descentralización de la toma de decisiones y una construcción de abajo a arriba, nos parece lo más conveniente.

Enfoque: Construcción de experiencias de democracias participativas. Acción: Articulación de grupos de trabajo, que ponen en marcha de forma coordinada sus propios proyectos. Productos: Ejecución a corto plazo de algunas propuestas, e inicio de otras a medio plazo.

\subsection{Los enfoques transductivos y los desbordes necesarios.}

Los procesos nunca terminan, lo contrario tal vez lo piense un técnico desde su lógica de proyectos, pero no es así para la gente. Cuando se concreta la ejecución de algunas de las propuestas se abren nuevas cuestiones, porque los "planes" nunca se ajustan completamente a la realidad y menos con los imprevistos que siempre suceden. Los "desbordes" abren de nuevo los procesos. Tanto aquellos desbordes de la situación en sí, como de los propios grupos que se implicaron, como de los aprendizajes de quienes están atentos a lo que se innovó en los conocimientos y las metodologías. La realidad es siempre algo abierto y sólo en ocasiones la podemos cerrar un poco, para poder entender algo, pero se abre de nuevo.

El octavo salto diferencial tiene que ver con la evaluación y monitoreo de los procesos que acompañamos. Esto va unido al tema anterior de la auto-organización, pues no se trata de una evaluación final, por la razón recién apuntada, y la mayor diferencia que vemos es la capacidad de "desborde" de los procesos, tanto para las propias administraciones, como para los sectores implicados, como incluso para el propio grupo motor. Estos actores deben estar dispuestos a ser desbordados por las circunstancias locales, pues en caso de que no se de ese salto hay que desconfiar de que se haya tocado la realidad de verdad. En este aspecto volvemos al primer salto de la "auto-reflexión", es decir a volver a comenzar con cada proceso, pues las circunstancias externas siempre son cambiantes y muy complejas, y lo que no se puede es repetir los mismos esquemas una y otra vez. Quién no sienta que está aprendiendo en cada uno de los casos es que está aplicando mecánicamente una metodología sin reinventarla con la gente.

Enfoque: Estilos transductivos que se dejan desbordar. Acción: Monitoreo con dispositivos de corrección y mejora de procesos. Producto: Resultados visibles a corto plazo, que dan confianza para los programas a medio plazo. 


\section{PLANES INTEGRALES DE BARRIOS Y PUEBLOS: EL PLAN INTEGRAL DE ENTREVÍAS-EL POZO}

Podemos apoyarnos en ejemplos de algunos Planes anteriores de barrios -como el Polígono Sur, en Sevilla; Palma-Palmilla, en Málaga; el distrito de Tetuán o el municipio de Zarzalejo en Madrid- o con otros ejemplos participativos con los que hemos colaborado en La Laguna o Lanzarote, en barrios de Barcelona o de Badalona, de A Coruña, Valencia o Mondragón, etc. Pero vamos a centrarnos en un Plan que está en marcha y que puede servir de referencia: sobre todo por sus dificultades.

El barrio de Entrevías-El Pozo ${ }^{6}$ es uno de los que forman el famoso distrito madrileño de Puente de Vallecas, que cuenta con una ejemplar historia en el campo del asociacionismo vecinal y de las luchas por la vivienda, la cultura, los equipamientos ciudadanos y en general el bienestar para sus habitantes ${ }^{7}$. Vallecas, y en concreto el barrio de Entrevías, es un referente dentro del movimiento vecinal, pero también es un territorio en el que perduran las desigualdades socioeconómicas con respecto a otros sectores de población y con otros barrios de la ciudad.

El asociacionismo vecinal nació en estos barrios y aún perdura. Sin embargo, no son suficientes estos elementos de movilización cuando se desata una crisis como la de 2008, cuando el retroceso de los derechos de la población ha sido tan importante. Tampoco son suficientes los planes de actuación de los dispositivos de las administraciones, con frecuencia carentes de un sentido comunitario, ni los numerosos proyectos que las entidades sociales llevan a cabo, a veces poco coordinados, cuando no solapados.

\subsection{El Inicio del Plan: el Grupo motor y los mapeos (saltos 1, 2, 3 y 4)}

Todo proceso social parte de lo ya vivido y en el caso que aquí presentamos su origen más inmediato arranca de un encuentro de entidades del barrio que tiene lugar en una de las Asociaciones de Vecinos (AA.VV.), la de El Pozo, hacia finales de 2015. En ella se muestra la desazón por la situación del barrio y se propone elaborar un Plan Integral. Esta Negociación Inicial (salto 2) cuenta con las AAVV, pero aún no con el Ayuntamiento. Se parte de una coyuntura en la que el nuevo Gobierno local tiene buenas relaciones con el tejido asociativo. Además, un grupo de jóvenes profesionales llevaban dos años con la Fundación CREASVI, formándose (salto 1) en metodologías socio-praxicas, lo que incluyó

${ }^{6}$ El nombre de Entrevías no sólo es una descripción del barrio, sino que construye el imaginario de cómo las vías del tren lo limitan, aíslan y coartan, aunque también lo comunican con la ciudad. El Pozo evoca aquellas tierras de cultivo y éstas sobre las que se fueron levantando casitas y, por fin, parte de la ciudad. Aunque administrativamente es un solo barrio, en ocasiones el habla de los habitantes puede diferenciar Entrevías de El Pozo.

7 Un documento imprescindible de cómo fue el proceso de conquista de la ciudad y construcción de ciudadanía, en casi una treintena de barrios de Madrid, es el de Villasante et. al., (1989) 
auto-reflexión, derivas, sociogramas y otras actividades previas al comienzo de esta fase.

De las primeras técnicas empleadas, para hacer un diagnóstico situacional, fue la elaboración de un mapa de los actores del barrio, un sociograma ${ }^{8}$, identificando y debatiendo sobre las relaciones existentes, la agrupación de algunos de estos actores en conjuntos de acción y las fracturas presentes en las redes. En ese momento (salto 3) apenas se muestran relaciones de conflicto entre el tejido asociativo, pero sí aparece una brecha entre la población paya y la de etnia gitana, así como conflictos vecinales, que se manifiestan de manera virulenta por la presencia en algunos pisos, ocupados para este fin, del tráfico de droga y la prostitución.

\section{Gráfico 1. Mapeo de actores del barrio (sociograma).}

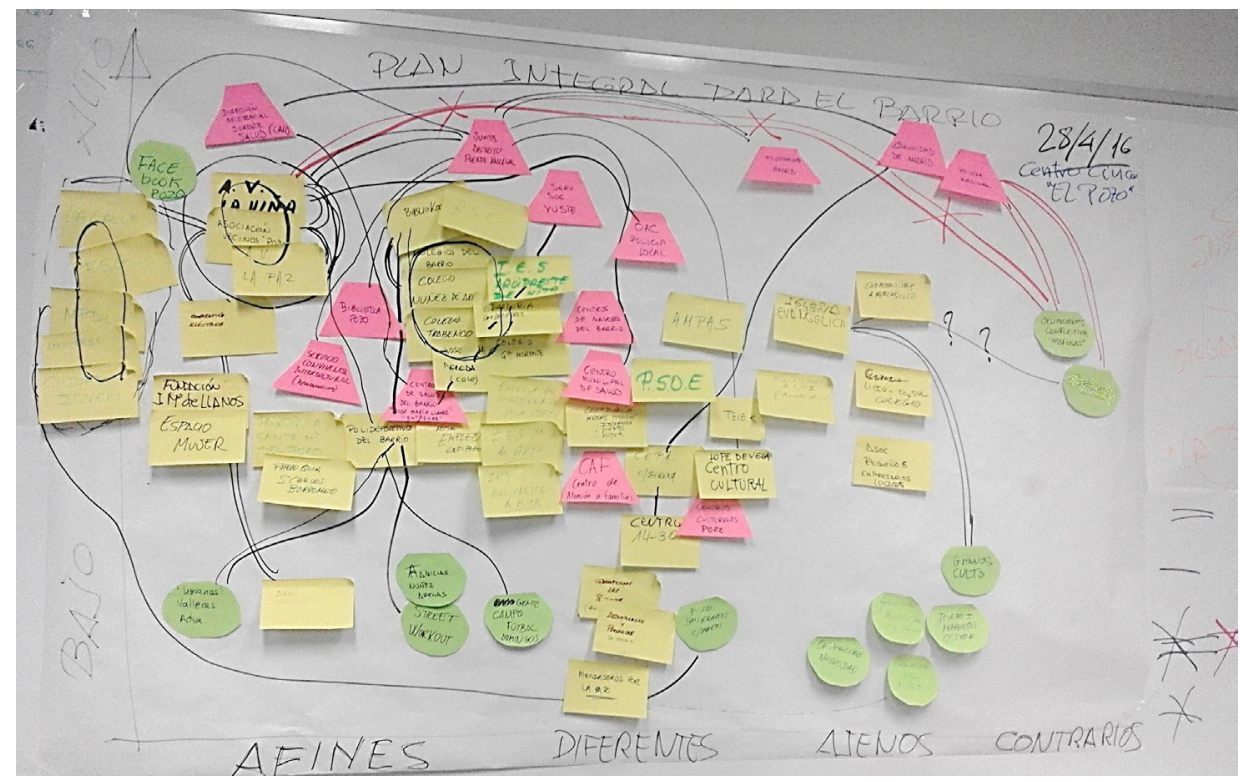

Fuente: Plan Integral ENTREPOZOyVÍAS

Tras este mapeo estratégico del grupo motor que se ha constituido se plantea completar un auto-diagnóstico que muestre, de la manera más concreta posible, aquellas demandas de la población y que sirva para actuar colectivamente. Pero no se trata de hacer un informe más, del tipo de los diagnósticos que abarrotan los cajones de las administraciones, sino un auto-diagnóstico situado, con las carencias percibidas y las demandas concretas de la población participante. La

8 Vid. Red CIMAS (2015), Villasante y Martín (2007), Villasante et. Al. (1989) y Martín (1999). Como referencia de otros manejos de los mapeos vid. Iconoclasistas (2013). y Botello et. al. (2013). 
manera de elaborarlo es a través de grupos de trabajo, coordinados por tareas, como la comunicación, el trabajo de calle, la documentación sobre los distintos Planes que afectan al barrio, etc.

Además, se opta por mapear sobre un plano de calles y parques, tanto las situaciones problemáticas (infraestructuras y su mantenimiento, limpieza, medioambiente, seguridad, empleo, salud, etc.) como las potencialidades (convivencia, solidaridad, espacios de encuentro...) para poder mostrar las posibilidades de la población a la hora de participar en la transformación de las situaciones indeseadas. Esto supone algún cambio en movilización convencional, pasando de la reivindicación a la participación directa, de la protesta a la propuesta y de la delegación a la actuación.

La técnica de este "mapeo geográfico" (salto 4), que consideramos una tecnología apropiada al caso. En síntesis, consiste en ubicar sobre un mapa del territorio aquellas situaciones problemáticas o las potencialidades que las personas participantes consideran relevantes. El resultado no será sólo el mapa con los contenidos iniciales, sino todo el debate y los aportes que se elaboran durante el tiempo que dura esta dinámica. La manera de recoger estos materiales puede ir desde el registro discursivo en audio o video al registro escrito o mediante dibujos, muy apropiado en el trabajo con niños. Estas fuentes primarias son la materia de análisis, que sin duda han de pasar por las reflexiones colectivas en posteriores encuentros.

En este caso los mapeos se realizaron en espacios abiertos del barrio, como la estación del tren de Cercanías o el parque forestal, aprovechando un concierto de hip-hop que atrajo a numerosos jóvenes del barrio y alrededores. También se hicieron mapeos con grupos más homogéneos y focalizados, como en los Centros de Salud, de Servicios Sociales, el Instituto o en colegios. Con esto se quería llegar a cuantos más sectores de la población, en un intento por captar sus demandas para un Plan de acción integral y con una proyección comunitaria. 


\section{Gráfico 2. Mapeo de problemas y potencialidades del barrio}

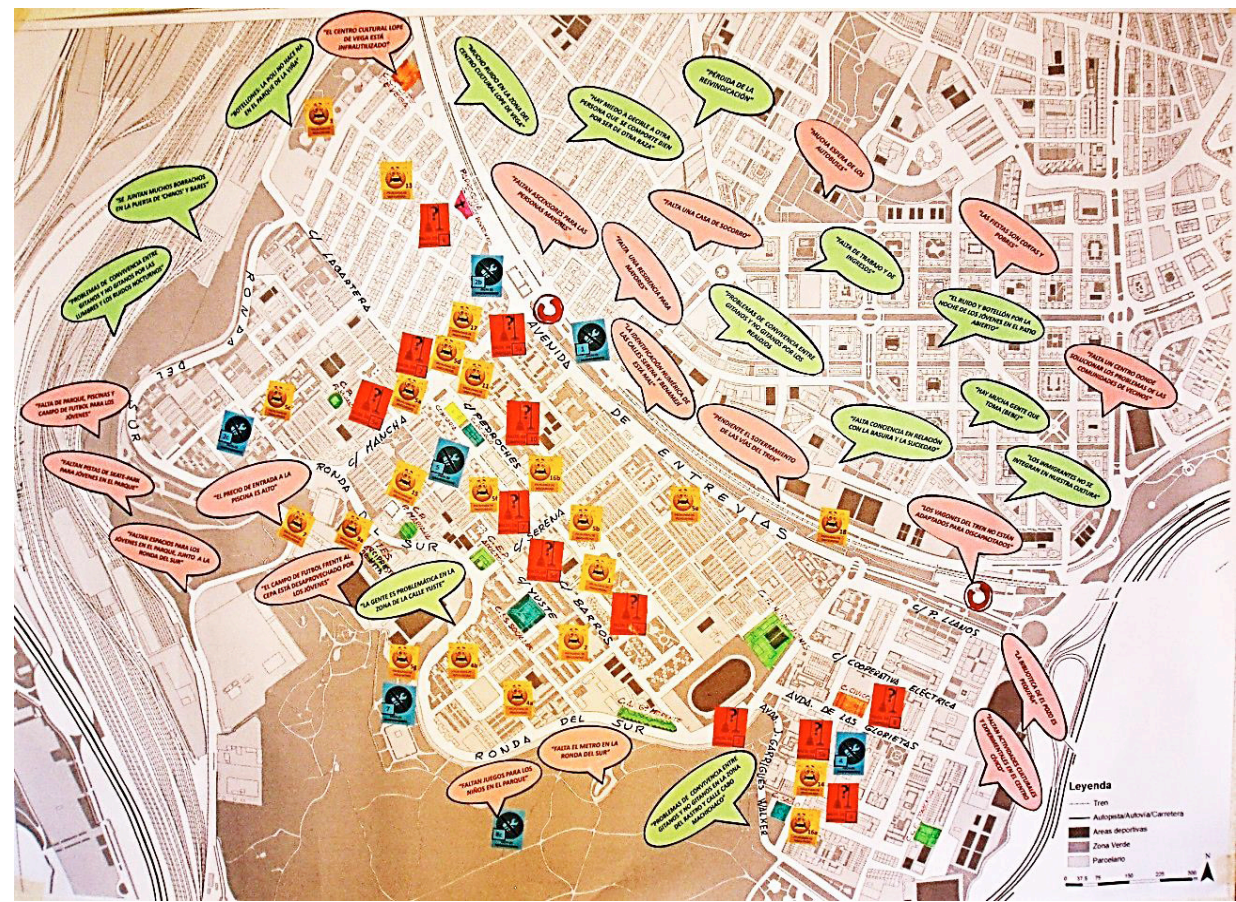

Fuente: Plan Integral ENTREPOZOyVÍAS

\subsection{Devolución de la información: reflexividad cooperativa (salto 5).}

Hemos hablado de la necesaria reflexión que requiere esta primera información en bruto y así se hizo posteriormente. En la primavera de 2017 se organizó un "Primer encuentro vecinal y de entidades ENTREPOZOyVÍAS" ${ }^{9}$ en el instituto Arcipreste de Hita, haciéndolo coincidir con la convocatoria para divulgar sus actividades con los estudiantes.

Los pasacalles, con una charanga-batucada, aportaron el carácter festivo a la jornada; los clásicos carteles anunciadores, la difusión entre las asociaciones, dispositivos y núcleos activos del barrio, junto con la chocolatada de rigor, hicieron que la asistencia fuera suficiente como para que se pudieran discutir la

${ }^{9}$ Nuestro grupo motor inicial ya tenía nombre gracias a la creatividad de una compañera, que propuso el de Colectivo "ENTREPOZOyVÍAS". 
información, que habíamos ido sistematizando en mapas-resumen por categorías y que estaban expuestos a la entrada del instituto.

La reflexión sobre lo ya dicho permitió concretar lo abstracto, profundizar lo superficial y, sobre todo, complejizar lo más simple. Es decir, se validó la información recogida, se profundizó en algunos temas de mayor interés, se priorizaron las actividades que se vieron capaces de poner en marcha, etc. En suma, se propuso una "reflexividad de segundo orden".

Se puede considerar que el tiempo transcurrido desde el comienzo hasta llegar a este punto, en el que todavía no estaba siquiera elaborado el Plan, es de una tremenda dilación. Pero es preciso reflexionar sobre los distintos tiempos que se viven en un barrio: el administrativo, el comunitario, el técnico, el asociativo, etc. Cada lógica cuenta con su reloj y su calendario y en nuestro caso íbamos al paso que los recursos y el resto de los tiempos nos marcaban, porque no es igual tener una jornada laboral dedicada exclusivamente al Plan que dedicarle el tiempo en el que coinciden las agendas de las personas que participan. Aunque se intentó en una negociación con el gobierno local (salto 2), en el grupo motor se sigue con la participación voluntaria de algunos profesionales de la salud, de la dinamización y de algunas entidades sociales y Asociaciones de Vecinos.

Pero además hay que tener en cuenta que el Plan no ha sido la única actividad que el grupo motor ha ido llevando a cabo, porque, a la vez que se iban realizando las actividades que aquí se describen, también se iba participando en las que la vida del barrio demandaba, como la organización de las fiestas (que las Asociaciones de Vecinos decidieron que debían cambiar y ser más comunitarias y participativas), el "Entrencuentro" (actividades de calle para niños organizadas por una veintena de entidades, dispositivos y grupos del barrio), los diferentes planes y espacios de participación propuestos por las administraciones (Foros Locales, Presupuestos Participativos, Plan Director para el Parque, Ruta walking people -WAP-, Plan Director para espacios públicos, etc.), además de las convocatorias que las urgencias del día a día demandaban.

\subsection{Coordinación de las redes e integralidad de las acciones (salto7).}

Como decíamos anteriormente los planes de intervención en el barrio son numerosos, aunque con frecuencia adolecen de falta de coordinación, de participación de la población o de evaluación de los resultados y los cambios producidos. El abordaje de estas deficiencias está entre los objetivos del Plan, el que las acciones sirvan tanto para cambiar las problemáticas que aborda, como para cambiar la organización de los actores del barrio. Para ello se ha venido trabajando con énfasis en la coordinación con otros grupos, entidades y dispositivos de las administraciones que son del barrio y/o están presentes en él.

Se viene haciendo un seguimiento de todos los planes desde las administraciones, viendo cómo se pueden coordinar estas acciones, por lo general carentes de un planteamiento participativo eficaz, con las que van surgiendo y se van plasmando en el Plan Integral. Pongamos un ejemplo: hay un proyecto de crea- 
ción de un huerto urbano comunitario, que se piensa como un espacio donde pueden confluir las actividades formativas del Centro de Educación de Personas Adultas (CEPA) con las de la Asociación de Asperger, las Asociaciones de Vecinos, el Instituto y los colegios, entre otras; pero también que sea un proyecto educativo para las personas participantes y para el barrio, un espacio de encuentro y convivencia, generador a su vez de otros proyectos (de economía social y solidaria, autoempleo, soberanía alimentaria, agroecología, etc.) y un proyecto saludable, como un nuevo activo de salud. Este enfoque integral no sólo potencia un amplio conjunto de acción de actores, sino que atraviesa numerosos ejes temáticos y existenciales del barrio y sus habitantes.

Sin embargo, llegado a este punto, el Plan no pretende ser asumible sólo por el grupo motor, ni tampoco por el conjunto asociativo del barrio o por algunos de los dispositivos de las administraciones públicas implicados. Es necesario que se dé una conjunción más abierta y diversa, un amplio conjunto de acción, que sea capaz de actuar y de desencadenar un círculo virtuoso en las condiciones de vida de este territorio. Por ello también es necesario priorizar aquellas acciones que son más viables también para los sectores no organizados. La priorización ya viene acompañada de un inicio de la organización de carácter democrático a la que nos referimos, una red conjuntada ${ }^{10}$ de actores orientados hacia el Plan. Si el grupo motor -con sus profesionales y asociaciones- asumiera en solitario llevar adelante el Plan reproduciría el mismo esquema que critica: un plan más que pretende ser la solución a los problemas del barrio, pero desvinculado del resto de planes y organizaciones. Es decir, crearía una nueva red centralizada y aislada.

\subsection{El Plan negociado y la idea-fuerza (salto 6).}

Con posterioridad a las actividades ya descritas se realizaron unos talleres para priorizar y concretar las acciones del Plan, así como para elaborar la IdeaFuerza. La comunicación para la convocatoria no se remitió exclusivamente al mailing, la cartelería o las llamadas telefónicas, sino que se hizo a modo de encuesta participativa, a través de visitas y entrevistas personales a un buen número de organizaciones y dispositivos del barrio, informándonos de qué hace cada entidad e informando a la otra parte de qué hacemos como grupo motor. Si tratamos esta estrategia comunicativa desde la perspectiva reticular podemos decir que se alcanzaron varios objetivos: se crearon vínculos de confianza con los actores más alejados, se reforzó la relación con quienes están más próximos y se saltó de unas redes más conocidas y frecuentadas a otras más desconocidas o distantes. Los puentes locales que se van creando permiten que se amplíe el conjunto de acción.

10 Cuando mencionamos la "red conjuntada" nos referimos a: "una elaboración propia de la gente a partir de que la red distribuida pura no existe, y que lo que encontramos son agrupaciones o conjuntos de acción [...] el análisis concreto de la situación concreta es lo que sigue siendo necesario hacer para salir de las dualidades abstractas" (Villasante 2014: 66) 
De estos talleres salieron las acciones que se pueden llevar a cabo de manera inmediata, mediante la auto-organización local (como una campaña de limpieza y educación cívica o un blog con calendario conjunto de convocatorias), las que requieren de más amplias alianzas y de algunos recursos con los que no se cuenta (el mencionado huerto comunitario o el seguimiento de las acciones del Plan Director para espacios públicos) y acciones que remiten a la reivindicación y la participación ante las administraciones u otras instancias (lo relativo a las grandes infraestructuras). Cada una de estas propuestas requiere a su vez de un grupo motor inicial y para concretar las acciones se utilizó una plantilla como la siguiente:

Cuadro 2 Tabla de planificación.

\begin{tabular}{|c|c|c|c|c|c|}
\hline OBJETIVOS & $\begin{array}{c}\text { PROPUESTA/ } \\
\text { ACCIÓN }\end{array}$ & $\begin{array}{c}\text { ACTORES } \\
\text { RESPONSABLES }\end{array}$ & $\begin{array}{c}\text { RECURSOS - } \\
\text { POTENCIALIDADES }\end{array}$ & CALENDARIO & $\begin{array}{c}\text { INDICADORES DE } \\
\text { CAMBIO }\end{array}$ \\
\hline & & & & & \\
\hline
\end{tabular}

Fuente: Plan Integral ENTREPOZOyVÍAS.

Otro elemento que se trató en estos últimos talleres, pero que sin duda requiere de más elaboración, es el de los indicadores de seguimiento y evaluación de las acciones, que no son sólo índices cuantitativos de difícil elaboración, sino que han de estar siempre al alcance de la mano de las personas y organizaciones participantes. Siempre hemos de tener en cuenta que aquellas acciones que desarrollen las personas participantes han de contar una tecnología que sea apropiable por los participantes, para que cree autonomía y no dependencia de técnicos, académicos, empresas o cualesquiera otros actores.

\section{AVANZANDO ALGUNAS CONCLUSIONES: DESBORDES Y EL OCTAVO SALTO.}

Vamos a presentar unas conclusiones provisionales que relacionen todo lo que hemos escrito sobre nuestros casos prácticos, sacando enseñanzas para las propias metodologías y también en relación con las democracias de iniciativas transformadoras de la sociedad en que nos toca vivir. Se discute sobre sus aplicaciones (Planes Integrales, comunitarios y otras metodologías) para hacer "democracias participativas" en ciudades, barrios y pueblos, pero habría que empezar por evaluar la complejidad de los procesos y no pensar que las comunidades o sectores poblacionales son unidades estancas con una sola dimensión y opinión. 
Estos procesos tienen su dificultad, y sobre todo parten de una desconfianza de la población, no solo hacia las administraciones, sino también hacia muchas asociaciones que se dicen representativas. Aprender de los errores propios y de los ajenos es una asignatura que aún no hemos desarrollado bastante, a pesar de que planes de tipo comunitario se llevan haciendo desde hace décadas.

Si un plan de barrio, por ejemplo, se basa en que una Junta Directiva que lleva años en la lucha local, presenta una lista de prioridades a las Administraciones, y alguna de éstas le concede unas ayudas, esto no es lo que aquí estamos diciendo que es un Plan Integral. Pero si esa asociación se junta con otras entidades, colectivos grupos de vecindario del barrio, etc., y aplica una serie de pasos y fases de escucha activa en los lugares de vida cotidiana de la gente; y luego hace talleres de devolución creativa y priorización; pasa a redactar un Plan, al tiempo que pone en marcha una propuesta de urgencia; y se auto-organiza en grupos de trabajo y monitoreo, ya estamos hablando de procesos más en serio. Estaremos construyendo "escuelas de democracia", de formación y acción desde abajo, descentralizadas y un futuro para el barrio o pueblo.

La planificación es distinta de los presupuestos, y en buena lógica debería preceder a las decisiones que se tomen año a año para las inversiones. En algún caso, como Badalona, hemos acompañado un proceso donde la Planificación a varios años (PAM), se hizo al tiempo que un Presupuesto Participativo, de forma que se pudieran retroalimentar en ambas direcciones. Un presupuesto anual que no tenga una planificación previa de mayor alcance puede ser un problema, incluso para tener en cuenta los "criterios" que deben ser anteriores a la priorización por la población. Para hacer bien un proceso participativo la planificación descentralizada por barrios (o por pueblos) puede ser de mucha utilidad, de forma que se puede ir conjuntado en niveles más amplios (por ejemplo, a nivel distrito, ciudad, comarca, etc.) y además verificando cada año con los presupuestos participativos, que actualicen las urgencias de cada momento.

Si queremos avanzar hacia democracias de iniciativas de base, de tipo transformador de la realidad existente, hay unas experiencias locales y unas bases metodológicas sobre las que se pueden ir construyendo nuevas realidades. Pero todo esto no llega por la proclama de un político o un dirigente social o un experto en la materia, sino por la construcción colectiva con grupos motores para tareas concretas viables en las que pueda creer la gente en su vida cotidiana. Los Planes integrales son una buena base para empezar, pero necesitan de metodologías, en las que hay que capacitarse, y no solo buena voluntad o una sucesión de técnicas. La gente no está deseando participar, sino que está muy desconfiada, y se necesitan pruebas y métodos probados que devuelvan el protagonismo a la población acostumbrada a la delegación y el clientelismo. Enfrentarse a esta inercia es sin duda una tarea imposible, pero, por eso mismo, necesaria. 


\section{BIBLIOGRAFÍA:}

BOTELLO, B. et al. (2013): "Metodología para el mapeo de activos de salud en una comunidad", en Gaceta Sanitaria, 27(2), pp. 180-183.

CALLEJO, J. y VIEDMA, A. (2006): Proyectos y estrategias de investigación social: la perspectiva de la intervención. Madrid, Ed. Mc Graw Hill.

CALLEJO, J. y VIEDMA, A. (2015): "La investigación-acción participativa", en M. García Ferrando, F. Alvira, L. E. Alonso y M. Escobar (coord.), El análisis de la realidad social: métodos y técnicas de investigación. Madrid, Alianza Editorial, pp. 295-328

CEA D'ANCONA, Ma A. (2012) Fundamentos y aplicaciones de la metodología cuantitativa. Madrid, Ed. Síntesis.

CEMBRANOS, F. y MEDINA, J. A. (2003): Grupos inteligentes: teoría y práctica del trabajo en equipo, Madrid, Ed. Popular.

COLECTIVO IOÉ (1989): "Participación ciudadana y voluntariado social". Disponible en: http://www.colectivoioe.org/uploads/42fba7f316a2add141626bc7e01f4ebe1321 c62b.pdf (Consulta: 27-05-2019).

COLECTIVO IOÉ (1996): "Voluntariado y democracia participativa. Reflexiones a partir del Proyecto +60, Investigación Acción Participativa en el barrio de Prosperidad (Madrid)". Disponible en: https://www.colectivoioe.org/uploads/62145a362f7e 337cbdbdc6e873e991146cf40b1d.pdf (Consulta: 27-05-2019)

CONTI, A. (2004): "La encuesta hoy. De la «coinvestigación» obrerista al «caminar preguntando» y más allá: la encuesta sobre las «formas de vida» en el «taller metropolitano del saber difuso»", en Malo, M. (coord.), Nociones comunes. Experiencias y ensayos entre investigación y militancia. Madrid, Ed. Traficantes de Sueños, pp. 43-53

GALTUNG, J. (2004): Transcender y transformar. Una introducción al trabajo de conflictos. México, Editorial Quimera

GUBA, E., \& LINCOLN, Y. (2002): "Paradigmas en competencia en la investigación cualitativa", en DENMAN, C. A., \& HARO, J. A. (coord.), Por los rincones. Antropología de los métodos cualitativos en la investigación social, Sonora-México, El Colegio de Sonora, pp. 113-145.

IBÁÑ̃Z, J. (1985): Del algoritmo al sujeto. Perspectivas de la investigación social. Madrid, Siglo XXI Editores

IBÁÑEZ, J. (2015) [1986]: "Perspectivas de la investigación social: el diseño en las tres perspectivas”, en M. García Ferrando, F. Alvira, L. E. Alonso y M. Escobar (coord.), El análisis de la realidad social: métodos y técnicas de investigación, Madrid, Alianza Editorial, pp. 31-66

ICONOCLASISTAS (2013): Manual de mapeo colectivo. Recursos cartográficos críticos para procesos territoriales de creación colaborativa. Buenos Aires, Tinta Limón. Disponible en: https://www.iconoclasistas.net/mapeo-colectivo/ (Consulta: 27-05-2019)

MARTÍN, P. (1999): "El sociograma como instrumento que desvela la complejidad", en Empiria, no 2, pp. 129-152.

MONTAÑÉS, M. (2007): "Más allá del debate cuantitativo/cualitativo: la necesidad de aplicar metodologías participativas conversacionales", Política y sociedad, 44(1), pp. 13-30. 
ORTÍ, A. (1986): "La apertura y el enfoque cualitativo o estructural: la entrevista abierta semidirectiva y la discusión de grupo", en M. García Ferrando, J. Ibáñez y F. Alvira (coord.), El análisis de la realidad social, Alianza Universidad Textos, Madrid, pp.156-186.

Red CIMAS (2015): Metodologías participativas. Sociopraxis para la creatividad social. Madrid, DEXTRA.

STANCHIERI, M. y ARICÓ, G. (2013): "La trampa urbanística de los vacíos urbanos: casos etnográficos en Barcelona", X Jornadas de Sociología, Facultad de Ciencias Sociales, Universidad de Buenos Aires, Buenos Aires. Disponible en: http://cdsa. aacademica.org/000-038/88.pdf (Consulta 27-05-2019)

VERCAUTEREN, D., MOUSS CRABBÉ, O., y MÜLLER, T. (2010): Micropolíticas de los grupos para una ecología de las prácticas colectivas, Madrid, Ed. Traficantes de Sueños.

VILLASANTE, T. (1994): "De los movimientos sociales a las metodologías participativas", en J. M. Delgado y J. Gutiérrez (coord.), Métodos y técnicas cualitativas de investigación en ciencias sociales, Madrid, Ed. Síntesis, pp. 399-424.

VILLASANTE, T. (1998): Cuatro redes para mejor-vivir. Del desarrollo local a las redes para mejor-vivir. Buenos Aires, Ed. Lumen / Humanitas.

VILLASANTE, T. (2007): "Una articulación metodológica: desde textos del Socioanálisis, I(A)P, F. Praxis, Evelin F. Keller, Boaventura S. Santos, etc.”, en Política y Sociedad, 44 (1), pp.141-157.

VILLASANTE, T. (2014): Redes de vida desbordantes. Fundamentos para el cambio desde la vida cotidiana. Madrid, Ed. La Catarata.

VILLASANTE, T. (2017): Democracias transformadoras: experiencias emergentes y alternativas desde los comunes, Barcelona, El Viejo Topo.

VILLASANTE, T., ALGUACIL, J.; DENCHE, C.; HERNÁNDEZ, A.; LEÓN, C.; Y VELÁZQUEZ, I. (1989). Retrato de chabolista con piso: Análisis de redes sociales en la remodelación de barrios de Madrid. Madrid, Cuadernos de Vivienda. IVIMA, SGV, Revista Alfoz-CIDUR.

VILLASANTE, T. y MARTÍN, P. (2007): "Redes y conjuntos de acción para aplicaciones estratégicas en los tiempos de la complejidad social", en Política y Sociedad, 44 (1), pp.125-140. 
